\title{
Full operator algorithm for hybrid simulation
}

\author{
Chung-Chan Hung and Sherif El-Tawil ${ }^{*, \dagger, \ddagger}$ \\ Department of Civil and Environmental Engineering, University of Michigan, Ann Arbor, MI 48109-2125, U.S.A.
}

\begin{abstract}
SUMMARY
One of the weaknesses of the operator splitting method (OSM) is that its corrector step employs the approximation that incremental forces are linearly related to the tested structure's initial stiffness matrix. This paper presents a new predictor-corrector technique in which the assumptions about the tested structure's response are shifted to the predictor step, which results in an enhancement in overall simulation accuracy, especially for nonlinear structures. Unlike OSM, which splits the displacement and velocity operators into explicit and implicit terms, the new method uses predicted accelerations to compute fully explicit displacement and velocity values in the predictor step. Another advantage of the proposed technique, termed the full operator method (FOM) is that its formulation makes it suitable for both quasi-static and real-time hybrid simulation. The effectiveness of FOM is first evaluated by investigating error propagation in an undamped single degree-of-freedom model. It is shown that the corrector step in FOM is able to significantly suppress aberrant simulation results caused by incorrect estimation of the structure's stiffness matrix. The performance of FOM is demonstrated by exercising two additional models, which exhibit significant inelastic behavior under the prescribed excitation. The simulation results show that the proposed FOM algorithm is capable of producing accurate solutions and that the corrector step is influential in effectively reducing simulation errors. It is also shown that FOM suppresses actuator displacement control errors because of its reliance on measured quantities in the corrector step. Copyright (C) 2009 John Wiley \& Sons, Ltd.
\end{abstract}

Received 19 November 2008; Revised 18 March 2009; Accepted 19 March 2009

KEY WORDS: hybrid simulation; tangent stiffness; corrector step; nonlinear model; dynamics; OpenSees; error control

\footnotetext{
*Correspondence to: Sherif El-Tawil, Department of Civil and Environmental Engineering, University of Michigan, Ann Arbor, MI 48109-2125, U.S.A.

†E-mail: eltawil@umich.edu

$\ddagger$ Professor.

Contract/grant sponsor: National Science Foundation; Contract/grant sponsor: CMS 0530383

Contract/grant sponsor: University of Michigan

Copyright (C) 2009 John Wiley \& Sons, Ltd.
} 


\section{INTRODUCTION}

The pseudo-dynamic test technique was first proposed by Takanashi et al. in 1978 [1] and has since become widely accepted as an effective approach for simulating structural behavior under earthquake loading. Hybrid simulation, a technique based on the pseudo-dynamic method, combines traditional computational modeling with physical testing to more generally simulate dynamic structural response. In this method, the entire structure is represented using a discrete mass system. The time-history response of the structure under seismic excitation is then computed by solving the differential equation of motion of the system using time-stepping integration methods. The difference between hybrid simulation and traditional computational simulation techniques is that some of the terms of the restoring force vector in the differential equation of motion are either directly obtained by measurement from a physical test or computed from a separate computational model running in parallel with the main numerical time integration scheme. When the entire restoring force vector is obtained from physical testing, hybrid simulation reverts back to the original pseudo-dynamic method. One of the key advantages of hybrid simulation is that it permits the integration of modeling and testing capabilities at different research sites.

Many explicit and implicit algorithms for pseudo-dynamic and hybrid testing have been proposed in the past three decades [2-14]. Implicit algorithms, which are unconditionally stable, are commonly preferred over explicit algorithms, which are generally limited by their intrinsic stability limit. Shing et al. [15] developed an implicit algorithm based on the implicit algorithm by Hilber et al. [16]. In this algorithm, a parameter was induced to control the value of the incremental displacement at each sub-step such that overshooting of the imposed displacement can be avoided. However, the accuracy of the scheme deteriorates if the estimated initial stiffness that is required during the simulation is incorrectly calculated.

Another implicit method, which has been widely adopted for hybrid simulation, is the operatorsplitting method (OSM) [17, 18]. The attractive feature of this method is the fact that it is effectively transformed into an explicit scheme when the tangent stiffness matrix of the structure, which is usually considered difficult to estimate during testing anyway, is replaced by the initial stiffness matrix. Consider the equation of motion of a discrete structural system:

$$
M a_{n+1}+C v_{n+1}+r_{n+1}=f_{n+1}
$$

where $M$ is the mass matrix of the structure, $C$ is the damping coefficient matrix, $r$ is the restoring force vector, $f$ is the vector of external excitation forces. The displacement and velocity at the next time step, $n+1$, obtained using Newmark's integration scheme can be expressed as

$$
\begin{aligned}
& d_{n+1}=\left\{d_{n}+\Delta t v_{n}+\frac{\Delta t^{2}}{2}(1-2 \beta) a_{n}\right\}+\Delta t^{2} \beta a_{n+1} \\
& v_{n+1}=\left\{v_{n}+\Delta t(1-\gamma) a_{n}\right\}+\Delta t \gamma a_{n+1}
\end{aligned}
$$

where $d$ is the vector of nodal displacements, $v$ is the vector of nodal velocities, $a$ is the vector of nodal accelerations, parameters $\gamma$ and $\beta$ define the variation of acceleration over a time step, $\Delta t$ is the incremental time step, subscript $n$ denotes the current time step.

In OSM, the displacement and velocity equations from the traditional Newmark integration scheme are split into predictor, which are explicit terms, and corrector responses. The predictor 
responses are:

$$
\begin{aligned}
& \tilde{d}_{n+1}=d_{n}+\Delta t v_{n}+\frac{\Delta t^{2}}{2}(1-2 \beta) a_{n} \\
& \tilde{v}_{n+1}=v_{n}+\Delta t(1-\gamma) a_{n}
\end{aligned}
$$

The corrector responses are:

$$
\begin{aligned}
& d_{n+1}=\tilde{d}_{n+1}+\Delta t^{2} \beta a_{n+1} \\
& v_{n+1}=\tilde{v}_{n+1}+\Delta t \gamma a_{n+1}
\end{aligned}
$$

The procedure for implementing OSM starts off by calculating the predictor responses using the response values from time step $n$. The predictor responses are then imposed on the tested structure, and the resulting restoring force $\tilde{r}_{n+1}$ is measured. The measured restoring force and the initial stiffness of the system are used to calculate the acceleration at time step $n+1$. After that, the corrector responses are obtained using the acceleration. The entire system behavior can be acquired by repeating the above procedure.

OSM is unconditionally stable when the initial stiffness of the tested structure is greater than the tangent stiffness during the entire test [4]. On the other hand, OSM becomes conditionally stable when it is applied to a system that shows stiffening behavior. The key limitation of the method is that when the tested system becomes inelastic, the primary assumption of OSM that the tangent stiffness of the system can be replaced by its initial stiffness breaks down. Under such conditions, the accuracy of the simulated response will be reduced $[4,13]$.

A few attempts have been made to estimate the tangent stiffness during pseudo-dynamic and hybrid testing, although not always for the purposes of improving the accuracy of the time integration algorithm. Nakashima and Kato [18] estimated the tangent stiffness matrix to correct displacement control errors induced by the actuators. In their work they used the displacement and restoring force in sub-steps within a time step to estimate the required tangent stiffness. Thewalt and Roman [19] used the Broyden-Fletcher-Goldfarb-Shanno (BFGS) formula [20] to estimate the tangent stiffness to calculate energy errors during the pseudo-dynamic test. Carrion and Spencer [21] calculated the tangent stiffness based on the Broyden formula [22] to compensate for the delay in real-time hybrid testing. Hung and El-Tawil [13] proposed a strategy for estimating the tangent stiffness of a system during hybrid simulation based upon the premise that the specimen's tangent stiffness does not change substantially during the few steps preceding the current time integration step. By exploiting this assumption, they solved a set of equations to identify the full stiffness matrix for a multi-degree-of-freedom (DOF) test specimen and combined it with the traditional OSM to improve the accuracy of the time integration scheme.

From the above discussion, it is clear that implicit algorithms for hybrid simulation are desirable because they ensure unconditional stability of the numerical integrations. The use of a tangent stiffness matrix, as opposed to an initial stiffness matrix, is also beneficial for highly nonlinear problems to enable an accurate solution scheme without iterations. However, estimation of an accurate tangent stiffness matrix during testing is difficult. As a result, a method, which adopts not only a strategy for estimating a reasonably accurate stiffness matrix, but also an active self-correction to suppress errors resulting from inaccuracies in the estimated stiffness matrix, is desired. This paper presents a new method, termed the full operator method (FOM), for hybrid simulation which does 
that. As described later on, the tangent stiffness matrix herein is estimated using various algorithms, including BFGS [20, 23], Davidon-Fletcher-Powell (DFP) [23], Broyden [22, 23], Broyden Family [23], and SR1 [23]. The proposed scheme is validated through several numerical examples, some of which exhibit significant nonlinear behavior.

\section{PROPOSED ALGORITHM FOR HYBRID TESTING}

The equation of motion for the predictor step can be expressed as

$$
M \hat{a}_{n+1}+C \hat{v}_{n+1}+\hat{r}_{n+1}=f_{n+1}
$$

where $\hat{a}_{n+1}$ is the predictor acceleration vector, $\hat{v}_{n+1}$ is the predictor velocity vector, $\hat{r}_{n+1}$ is the predictor restoring force vector at time step $n+1$. Once the predictor acceleration vector is computed as discussed later on, the predictor velocity and displacement vectors can be obtained as

$$
\begin{aligned}
& \hat{d}_{n+1}=\left\{d_{n}+\Delta t v_{n}+\frac{\Delta t^{2}}{2}(1-2 \beta) a_{n}\right\}+\Delta t^{2} \beta \hat{a}_{n+1} \\
& \hat{v}_{n+1}=\left\{v_{n}+\Delta t(1-\gamma) a_{n}\right\}+\Delta t \gamma \hat{a}_{n+1}
\end{aligned}
$$

The predictor restoring force at time step $n+1$ can be approximated using the tangent stiffness as

$$
\hat{r}_{n+1} \approx r_{n}+\hat{K}_{n+1}^{\mathrm{T}}\left(\hat{d}_{n+1}-d_{n}\right)
$$

where $\hat{K}_{n+1}^{\mathrm{T}}$ is the estimated tangent stiffness at time step $n+1$, discussed later on in the paper. Substituting Equations (6) and (7) into Equation (5), the predictor acceleration at time step $n+1$ can be expressed as

$$
\begin{aligned}
\hat{a}_{n+1} & =\hat{M}_{n+1}^{-1} \hat{f}_{n+1} \\
\hat{M}_{n+1} & =M+\hat{C}_{n+1} \Delta t \gamma+\hat{K}_{n+1}^{\mathrm{T}} \Delta t^{2} \beta \\
\hat{f}_{n+1} & =f_{n+1}-\hat{C}_{n+1} v_{n}-\hat{C}_{n+1} \Delta t(1-\gamma) a_{n}-r_{n}-\hat{K}_{n+1}^{\mathrm{T}}\left[\Delta t v_{n}+\frac{\Delta t^{2}}{2}(1-2 \beta) a_{n}\right]
\end{aligned}
$$

where $\hat{M}_{n+1}$ is the predictor equivalent mass matrix; $\hat{C}_{n+1}$ is the predictor damping coefficient matrix, which is constant if it is mass-proportional or can be computed from $\hat{K}_{n+1}^{\mathrm{T}}$ if stiffness proportional damping is assumed; $\hat{f}_{n+1}$ is the predictor equivalent force vector. Equation (8) implies that $\hat{a}_{n+1}$ is a direct function of the estimated tangent stiffness $\hat{K}_{n+1}^{\mathrm{T}}$. In a manner similar to Equation (7), the restoring force relationship at time step $n+1$ is approximated as

$$
r_{n+1} \approx \hat{r}_{n+1}+\hat{K}_{n+1}^{\mathrm{T}}\left(d_{n+1}-\hat{d}_{n+1}\right)
$$

From Equations (6) and (9), the difference between the corrector and predictor restoring forces can be expressed as

$$
r_{n+1}-\hat{r}_{n+1}=\Delta t^{2} \beta \hat{K}_{n+1}^{\mathrm{T}}\left(a_{n+1}-\hat{a}_{n+1}\right)
$$


Equation (10) contains second-order terms of the incremental time step. These terms are also a function of the difference between acceleration vectors, each of which contains small acceleration values. Therefore, it is reasonable to assume that the difference between both vectors is negligibly small compared with either restoring force vector. In other words

$$
r_{n+1} \approx \hat{r}_{n+1}
$$

The numerical studies presented later on in the paper confirm that Equation (11) is an accurate assumption. An advantage of making the assumption in Equation (11) is that it shields the solution from the uncertainty introduced by using the tangent stiffness in the corrector step.

The corrector acceleration is calculated by substituting Equations (2) and (11) into (1). The resulting solution can be expressed as

$$
\begin{aligned}
a_{n+1} & =M_{n+1}^{\prime-1} f_{n+1}^{\prime} \\
M_{n+1}^{\prime} & =M+C_{n+1} \Delta t \gamma \\
f_{n+1}^{\prime} & =f_{n+1}-C_{n+1} v_{n}-C_{n+1} \Delta t(1-\gamma) a_{n}-r_{n+1}
\end{aligned}
$$

where $M_{n+1}^{\prime}$ is the corrector equivalent mass matrix, $f_{n+1}^{\prime}$ is the corrector equivalent force vector. $C_{n+1}$ is the corrector damping coefficient matrix assumed to be equal to the predictor value, i.e. $C_{n+1}=\hat{C}_{n+1}$. Unlike the predictor acceleration, the corrector acceleration is not associated with the estimated tangent stiffness but is computed from the measured restoring force from the tested structure.

The procedure of the proposed method is illustrated in Figure 1. First, the predictor displacement and velocity from Equation (6) are calculated using the predictor acceleration from Equation (8). The computed predictor displacement $\hat{d}_{n+1}$ and velocity $\hat{v}_{n+1}$ are imposed on the tested structure. The resulting restoring forces from the tested structure are then used in Equation (12) to calculate the corrector acceleration, which is then substituted into Equation (2) to generate the corrector displacement $d_{n+1}$ and velocity $v_{n+1}$. Meanwhile, the new estimated tangent stiffness $\hat{K}^{\mathrm{T}}$ for the next time step is computed using the measured increment in the predictor displacement $\Delta \hat{d}_{n}^{m}=\hat{d}_{n+1}^{m}-\hat{d}_{n}^{m}$ and restoring force vector $\Delta r_{n}=r_{n+1}-r_{n}$, as well as the current tangent stiffness; where $\hat{d}_{n+1}^{m}$ is defined as the measured predictor displacement $\hat{d}_{n+1}$. Since both the predictor displacement and velocity are generated from the algorithm, an advantage of this method is that it can be applied to real-time hybrid simulation.

The proposed FOM for hybrid simulation is viewed as an improvement over the regular OSM for two reasons. In the predictor step, the regular OSM, as shown in Equation (3), neglects the implicit term $a_{n+1}$ in the Newmark integration scheme. FOM increases the accuracy of the predictor by including this term and by relying on the estimated tangent stiffness as shown in Figure 1. Second, the corrector acceleration in OSM is based on the estimated initial stiffness of the system, which adversely affects accuracy when the tested structure exhibits significant inelastic behavior. In contrast, FOM employs the measured restoring force from the tested structure, without resorting to approximations such as those used in OSM. These two points suggest that FOM is a better candidate for general hybrid simulation, as it is demonstrated quantitatively later on in the paper. Since computation of the tangent stiffness during testing is important to the success of the proposed methodology, various techniques for computing it are introduced next. 


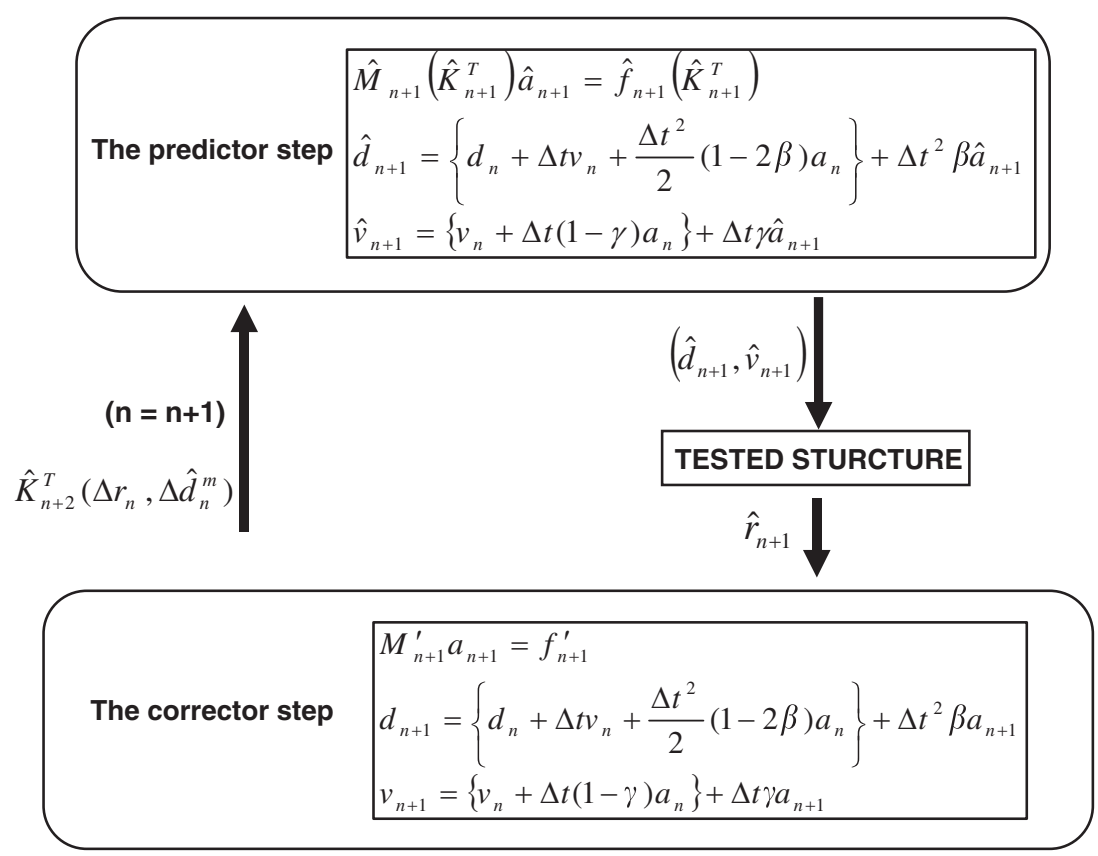

Figure 1. Algorithm for the proposed method.

\section{METHODS FOR ESTIMATING THE TANGENT STIFFNESS}

In this paper, five different algorithms, including BFGS, DFP, Broyden, Broyden Family, and SR1, are used to estimate the tangent stiffness. The first method, the BFGS method, which is considered the most effective [23] of all quasi-Newton updating formulae, is a method to solve for unconstrained nonlinear optimization problems. The BFGS formula for updating tangent stiffness is shown below [23]

$$
\hat{K}_{n+1}^{\mathrm{T}}=\hat{K}_{n}^{\mathrm{T}}+\frac{\Delta r_{n-1} \Delta r_{n-1}^{\mathrm{T}}}{\Delta r_{n-1}^{\mathrm{T}} \Delta \hat{d}_{n-1}^{m}}-\frac{\hat{K}_{n}^{\mathrm{T}} \Delta \hat{d}_{n-1}^{m}\left(\hat{K}_{n}^{\mathrm{T}} \Delta \hat{d}_{n-1}^{m}\right)^{\mathrm{T}}}{\left(\Delta \hat{d}_{n-1}^{m}\right)^{\mathrm{T}} \hat{K}_{n}^{\mathrm{T}} \Delta \hat{d}_{n-1}^{m}}
$$

where all the terms were previously defined. The DFP method is a quasi-Newton method that generalizes the secant method for multi-dimensional problems. The DFP formula for updating tangent stiffness is defined in Equation (14). Although DFP has been largely abandoned by many current researchers in favor of the BFGS method, the DFP method is still nevertheless used in this study as one of the candidate methods for updating the tangent stiffness

$$
\hat{K}_{n+1}^{\mathrm{T}}=\left(I-\frac{\Delta r_{n-1}\left(\Delta \hat{d}_{n-1}^{m}\right)^{\mathrm{T}}}{\Delta r_{n-1}^{\mathrm{T}} \Delta \hat{d}_{n-1}^{m}}\right) \hat{K}_{n}^{\mathrm{T}}\left(I-\frac{\Delta \hat{d}_{n-1}^{m} \Delta r_{n-1}^{\mathrm{T}}}{\Delta r_{n-1}^{\mathrm{T}} \Delta \hat{d}_{n-1}^{m}}\right)+\frac{\Delta r_{n-1} \Delta r_{n-1}^{\mathrm{T}}}{\Delta r_{n-1}^{\mathrm{T}} \Delta \hat{d}_{n-1}^{m}}
$$


Broyden's method, which was first documented by Broyden [22], is a quasi-Newton method for the numerical solution of nonlinear equations with multiple variables. When used for updating the tangent stiffness it can be expressed as

$$
\hat{K}_{n+1}^{\mathrm{T}}=\hat{K}_{n}^{\mathrm{T}}+\frac{\left(\Delta r_{n-1}-\hat{K}_{n}^{\mathrm{T}} \Delta \hat{d}_{n-1}^{m}\right)\left(\Delta \hat{d}_{n-1}^{m}\right)^{\mathrm{T}}}{\left(\Delta \hat{d}_{n-1}^{m}\right)^{\mathrm{T}} \Delta \hat{d}_{n-1}^{m}}
$$

Broyden's family is a linear combination of BFGS and DFP with a parameter controlling the weighting of BFGS and DFP contributing to the final solution. In this method, the positive definiteness of the Hessian approximations is also enforced [23]. Broyden's family algorithm used for estimating tangent stiffness is shown below, where the parameter $\psi$ is between 0 and 1 . In this paper, $\psi$ is chosen as 0.5

$$
\hat{K}_{n+1}^{\mathrm{T}}=(1-\psi) \hat{K}_{n+1}^{\mathrm{T}, \mathrm{BFGS}}+\psi \hat{K}_{n+1}^{\mathrm{T}, \mathrm{DFP}}, \quad \psi \in[0,1]
$$

The formula for updating tangent stiffness using the SR1 method is given in Equation (17). Unlike rank-two update methods (e.g. BFGS and DFP), the SR1 method does not guarantee that the updated tangent stiffness will maintain positive-definiteness. There is an evidence that SR1 often generates a better approximation than the BFGS method [23].

$$
\hat{K}_{n+1}^{\mathrm{T}}=\hat{K}_{n}^{\mathrm{T}}+\frac{\left(\Delta r_{n-1}-\hat{K}_{n}^{\mathrm{T}} \Delta \hat{d}_{n-1}^{m}\right)\left(\Delta r_{n-1}-\hat{K}_{n}^{\mathrm{T}} \Delta \hat{d}_{n-1}^{m}\right)^{\mathrm{T}}}{\left(\Delta r_{n-1}-\hat{K}_{n}^{\mathrm{T}} \Delta \hat{d}_{n-1}^{m}\right)^{\mathrm{T}} \Delta \hat{d}_{n-1}^{m}}
$$

In the following section, the effectiveness of the corrector step for suppressing inaccurate structural behavior resulting from an approximate or miscalculated tangent stiffness, i.e. as computed from one of the methods listed above, is numerically investigated.

\section{PERFORMANCE OF FOM}

In order to investigate the performance of FOM, an undamped single-DOF (SDOF) model with linear behavior is considered. In this numerical investigation, the external excitation is assumed to be zero. The system response is simulated using the proposed algorithm with an initial disturbance of a unit displacement. The estimated tangent stiffness is assumed to be $K_{\text {est }}=\lambda K_{\text {real }}$; where $K_{\text {est }}$ and $K_{\text {real }}$ are the estimated and real tangent stiffnesses of the model, respectively, $\lambda$ is the stiffness ratio of the estimated tangent stiffness to the real tangent stiffness. The real tangent stiffness, $K_{\text {real }}$, of the model is assumed to be $1 \mathrm{lb} / \mathrm{in}$.

The simulation procedure is illustrated in Figure 2. The predictor displacement is first computed using the assumed estimated stiffness and is then imposed on the tested model. The resulting reaction force is calculated by multiplying the predictor displacement with the real stiffness. After that, the corrector displacement is generated using Equations (2) and (5). The model response to the initial disturbance is then acquired by repeating the above procedure. Since there is no external excitation except the initial disturbance, the system oscillating with a constant unit amplitude is the analytic response. In this investigation, the system is characterized by using a parameter $\Omega$, defined as $\Omega=w_{n} \Delta t$, that accounts for the effect of step size and natural frequency on the solution; where $w_{n}$ is the natural frequency of the system, $\Delta t$ is the step size. Two different models with $\Omega=0.02$ and 0.1 are investigated. For each case, two different stiffness ratios, $\lambda=0.1$ and 10 , are 


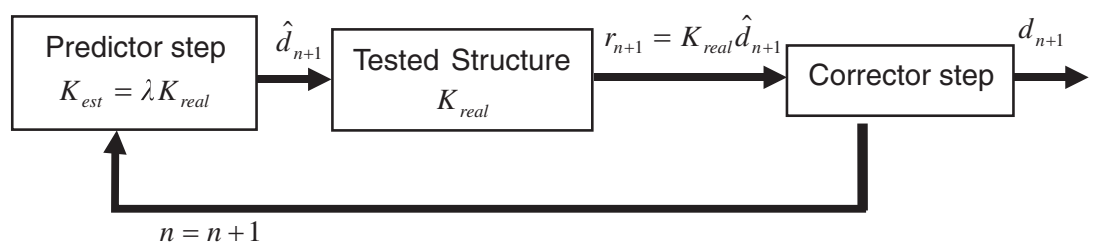

Figure 2. Procedure for investigation of the influence of the corrector step on the simulated solution.

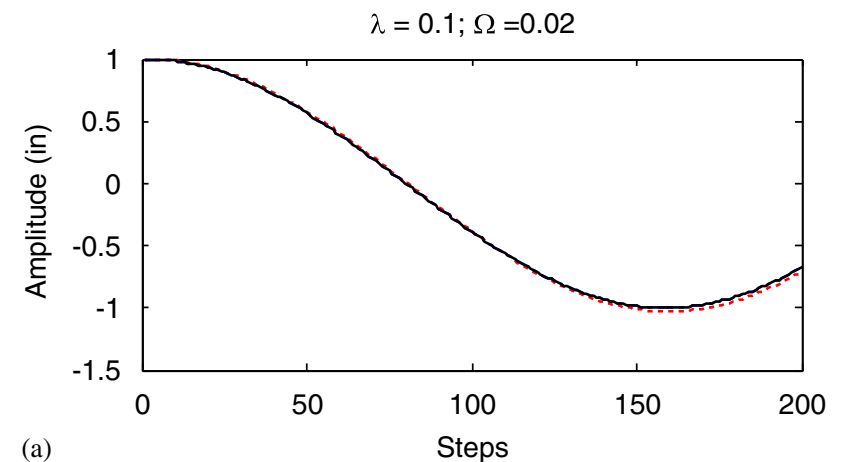

(a)

$$
\lambda=10 ; \Omega=0.02
$$

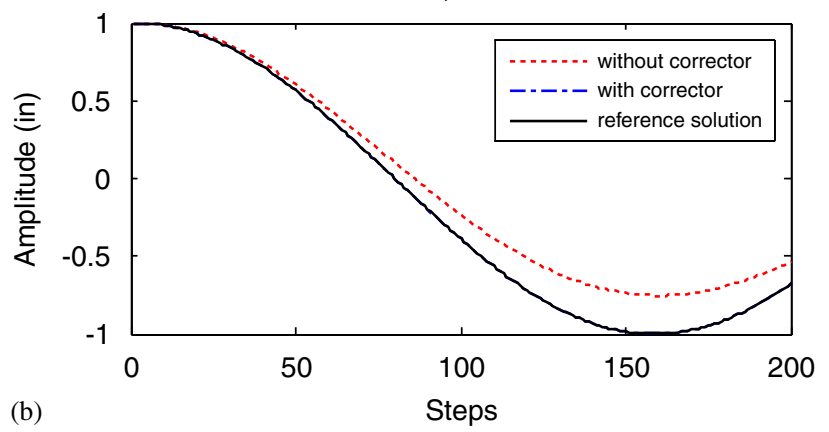

Figure 3. Model response with $\Omega=0.02$ using different stiffness ratios: (a) $\lambda=0.1$ and (b) $\lambda=10$.

considered. The simulated responses using the proposed method with and without the corrector step are compared with the reference solution from the traditional Newmark method with the correct tangent stiffness. The parameters $\gamma$ and $\beta$ in the integration algorithm are chosen to be $\frac{1}{2}$ and $\frac{1}{4}$, respectively.

The computed solutions from the system with $\Omega=0.02$ are plotted in Figure 3. It is observed from Figure 3(a) that, when $\lambda=0.1$, the proposed method produces accurate results compared with the reference solution whether the scheme incorporates the corrector step or not. In Figure 3(b), where $\lambda=10$, it is seen that employing the corrector step generates a better result than when the corrector is not used. On the other hand, as $\Omega$ is increased to 0.1 , the scheme without the corrector 

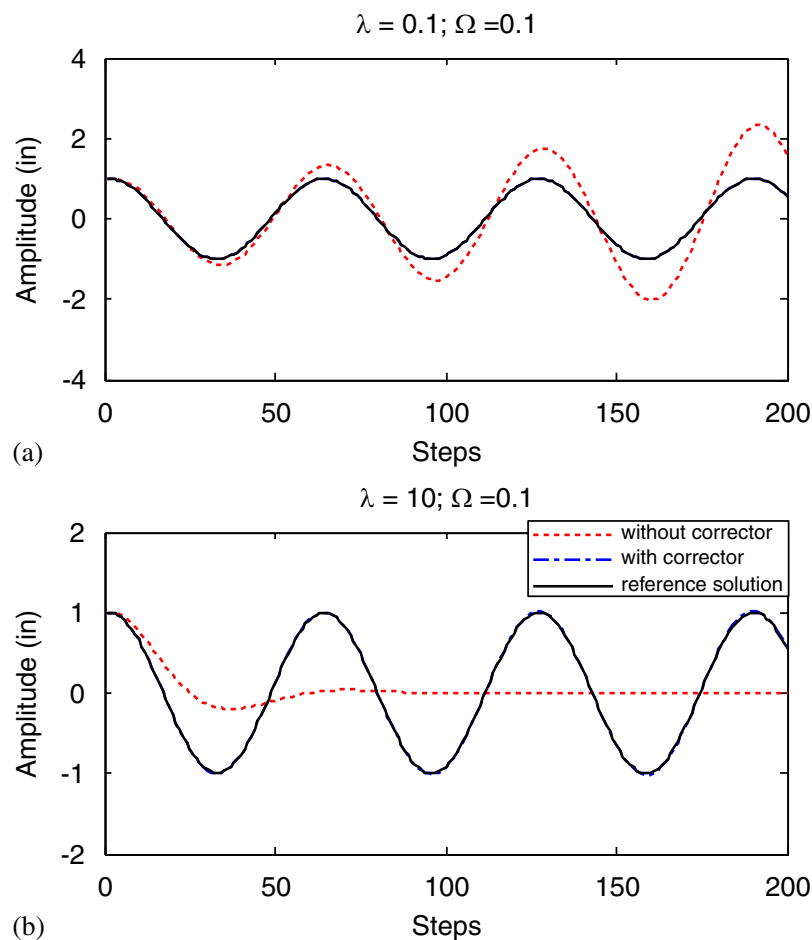

Figure 4. Model response with $\Omega=0.1$ using different stiffness ratios: (a) $\lambda=0.1$ and (b) $\lambda=10$.

step loses its ability to track the reference solution as shown in Figure 4. However, in contrast, the scheme with the corrector is still able to generate a result coinciding with the reference solution.

Comparing Figures 3 with 4, it is inferred that not using the corrector step produces results that are increasingly in error as $\Omega$ goes up. On the other hand, once the corrector step is employed, the displacement error is greatly diminished. Another observation is that for those results from the scheme without the corrector step, the oscillation amplitude becomes larger as time proceeds when the estimated tangent stiffness is less than the real tangent stiffness, whereas the amplitude diminishes with time when the stiffness ratio is larger than 1.

The cumulative energy error, $E_{\mathrm{c}}$, computed for the response for the various systems is plotted in Figures 5 and 6. The cumulative energy error is defined in [13] as

$$
E_{\mathrm{c}}=\sum_{j=1}^{j=n}\left|\left(r_{j}\right)_{\mathrm{OpenSees}}\left[\left(d_{j}\right)_{\mathrm{EFOM}}-\left(d_{j}\right)_{\text {OpenSees }}\right]\right|
$$

where $\left(d_{j}\right)_{\mathrm{EFOM}}$ is the displacement of node of interest computed from FOM, $\left(d_{j}\right)_{\text {OpenSees }}$ is the displacement of the node of interest from OpenSees, $\left(r_{j}\right)_{\text {OpenSees }}$ is the restoring force at the same node from OpenSees, and $n$ is the current time step. It is clear from both Figures 5 and 6 that $E_{\mathrm{c}}$ increases as $\Omega$ increases regardless of whether the corrector step is used or not. However, it can also be seen that deploying the corrector step significantly diminishes $E_{\mathrm{c}}$ suggesting that FOM can effectively maintain simulation accuracy even when the tangent stiffness is incorrectly estimated. 


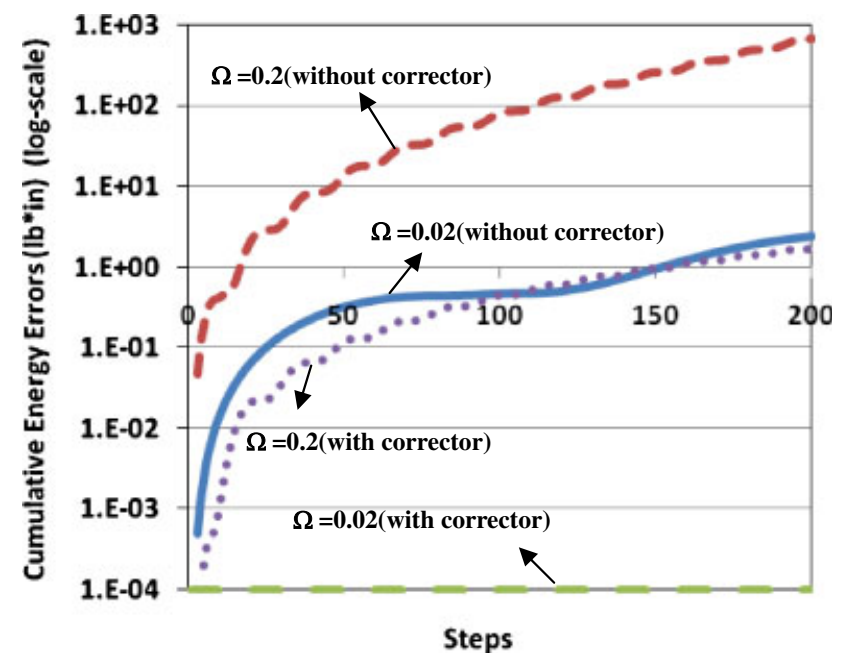

Figure 5. Displacement errors with and without the corrector step when $\lambda=0.1$.

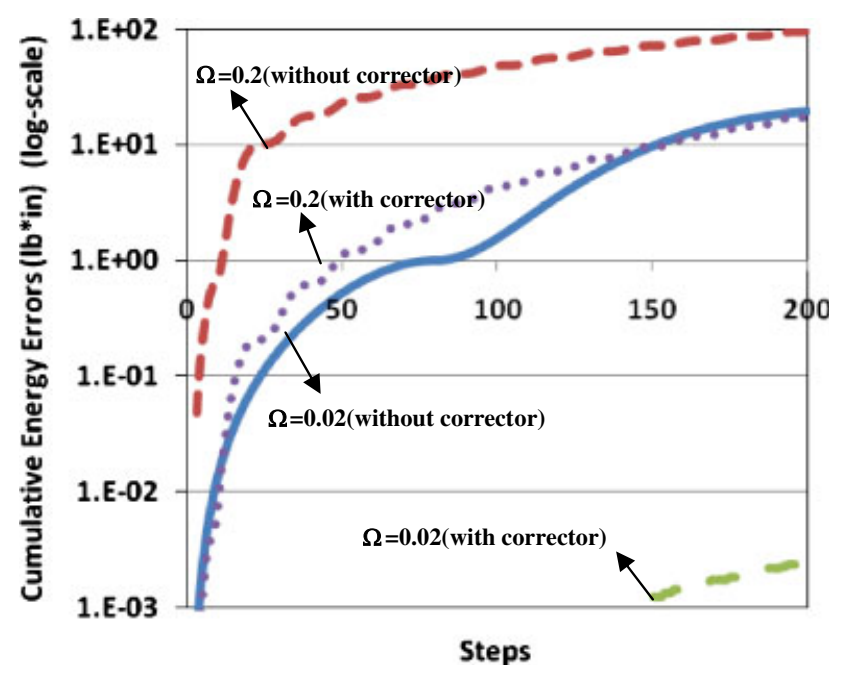

Figure 6. Displacement errors with and without the corrector step when $\lambda=10$.

\section{APPLICATION OF FOM TO NONLINEAR PROBLEMS}

Two numerical examples are used to demonstrate the capabilities of the proposed method when applied to nonlinear structures. The 1940 El Centro earthquake history record, scaled such that $\mathrm{PGA}=1 \mathrm{~g}$, is used as seismic excitation for both examples. UI-SimCor [24] is used as the platform for data transmission between the main computer and the substructures, which are numerically modeled using OpenSees [25]. The simulated responses using the proposed algorithm are compared with reference solutions from numerical simulations of the entire systems in OpenSees. The 


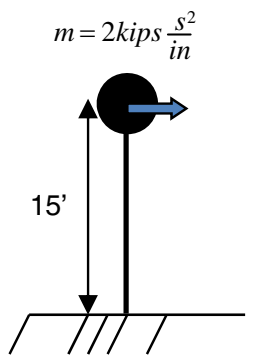

Figure 7. Properties of the shear wall model.

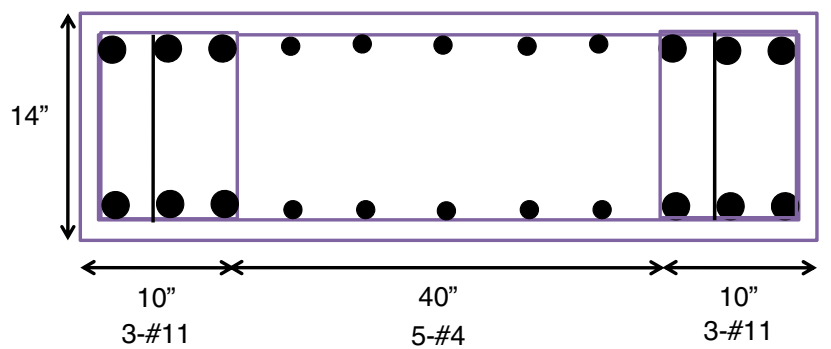

Figure 8. Cross section of the shear wall model.

parameters $\gamma$ and $\beta$ are chosen as $\frac{1}{2}$ and $\frac{1}{4}$, respectively. In order to show the effect of different schemes for estimating tangent stiffness on the model response, the tangent stiffness matrix is updated using the previously mentioned schemes, i.e. BFGS, DFP, Broyden, Broyden Family, and SR1.

Two rules recommended by Hung and El-Tawil [13] are imposed on the algorithm to ensure reasonable results. The first rule resets the reloading/unloading tangent stiffness to the initial stiffness right after the point where the displacement direction changes. Although this rule limits the proposed validation study to structures dominated by first mode response, where unloading of one member is associated with unloading of the entire structure, it does not necessarily limit the applicability of FOM to structures dominated by first mode effects. The second rule is more practical in nature. It filters out the steps where the imposed displacements are smaller than a user-specified threshold, specified as a minimum incremental displacement. This is to ensure that only steps that are large enough to contribute reliably to the stiffness calculations are utilized in the computations. Although user experience clearly plays a role in identifying this parameter, a number that is 2-3 times the displacement resolution of the actuator is recommended.

The first example is a reinforced concrete shear wall model as shown in Figure 7. The shear wall model is simulated as a 1 DOF structure with a mass of $2 \mathrm{kips} \mathrm{s}^{2} / \mathrm{in}$, and height of $15 \mathrm{in}$. Cross section details are shown in Figure 8. Concrete02 and Steel01 material models in OpenSees are used in the simulation and the associated parameter definitions are shown in Figure 9 and Table I. An incremental time step of $0.02 \mathrm{~s}$ is used for this example. To study the operation of FOM in detail and understand how its various components contribute to a successful solution, the following four schemes are simulated: (1) FOM with initial stiffness and without the corrector 


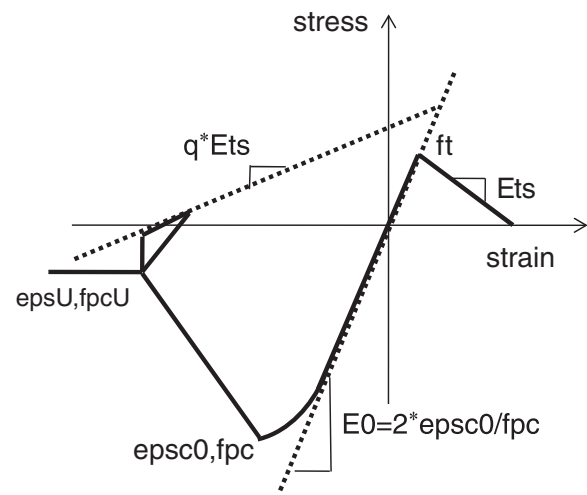

(a)

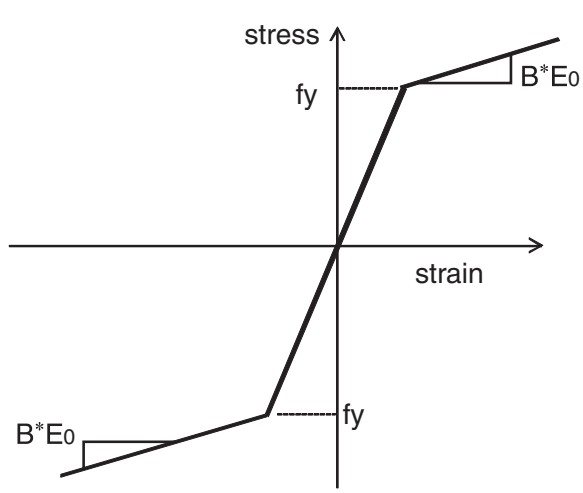

(b)

Figure 9. Concrete02 and Steel01 material models in OpenSees: (a) Concrete02 model and its parameters and (b) Steel01 model and its parameters.

Table I. Material parameters of the concrete and steel models.

\begin{tabular}{|c|c|c|c|c|c|c|c|}
\hline Concrete properties & $f p c(k s i)$ & epsc0 & $f p c u(k s i)$ & eps $U$ & $q$ & $f t(k s i)$ & $E t s(k s i)$ \\
\hline Unconfined concrete & -6 & -0.002 & -0.4 & -0.01 & 0.1 & 0.6 & 300 \\
\hline Confined concrete & -7.2 & -0.0045 & -2.4 & -0.03 & 0.1 & 0.72 & 360 \\
\hline \multirow[t]{2}{*}{ Steel bar properties } & $f y(k s i)$ & $E 0(k s i)$ & $B$ & & & & \\
\hline & 60 & 29000 & 0.01 & & & & \\
\hline
\end{tabular}

step, (2) FOM with initial stiffness and with the corrector step, (3) FOM with updated tangent stiffness and without the corrector step, and (4) FOM with updated tangent stiffness and corrector step, i.e. the full capability of FOM.

The simulation result from the first scheme is shown in Figure 10(a). It is clear that the resulting simulated behavior diverges from the reference solution when the corrector step and the updated tangent stiffness are not used. On the other hand, the solution improves greatly when the other three schemes are employed as can be seen in Figure 10(b). Although the figure only shows results from the BFGS updating method, equally good results are obtained when the other four updating methods are used. These are not shown so not to clutter up the figure.

The influence of the various parts of FOM can be seen in Figure 11, which plots $E_{\mathrm{c}}$ (on a $\log$ scale) computed from the four different schemes. Clearly, and as was obvious in Figure 10(a), using only the initial stiffness without the corrector step produces simulation errors that are much larger than those produced by the other three schemes. Using only the tangent stiffness matrix (without a corrector step) reduces $E_{\mathrm{c}}$ by approximately an order of magnitude, while incorporating the corrector step in both cases dramatically reduces $E_{\mathrm{c}}$. Using a tangent stiffness with a corrector step, which is the case when FOM is fully implemented, is the best situation. Figure 11 also demonstrates the advantage of FOM over OSM. In particular, OSM accumulates errors that are two orders of magnitude greater than the full implementation of FOM for this particular problem (Figure 11(a)). The superiority of FOM over OSM can also be seen in Figure 11(b), which shows 


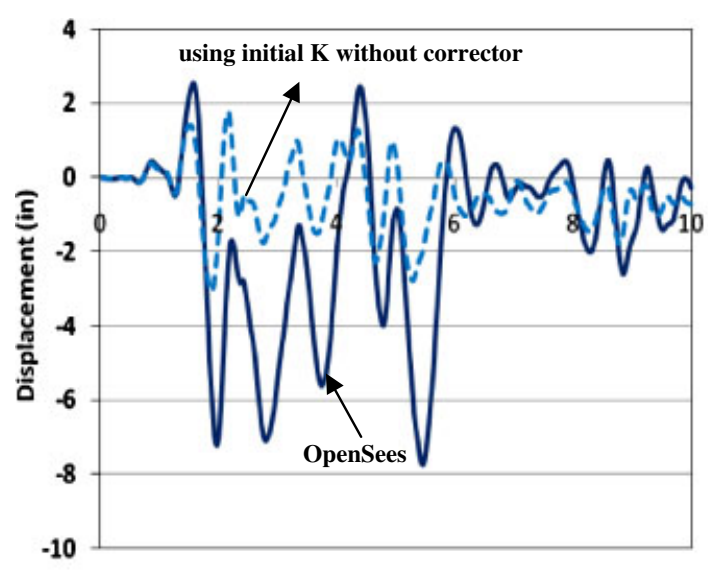

(a)

Time (sec)

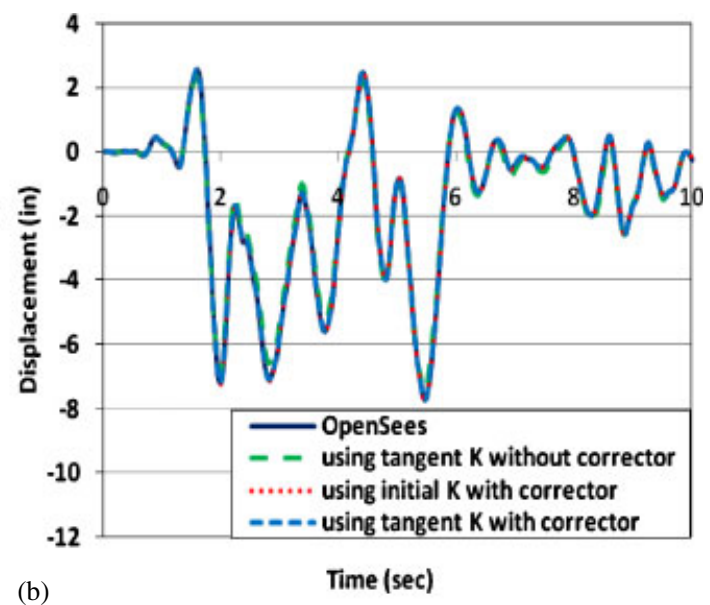

Figure 10. Simulated seismic responses of the shear wall model: (a) initial stiffness without the corrector step and (b) results from various FOM schemes.
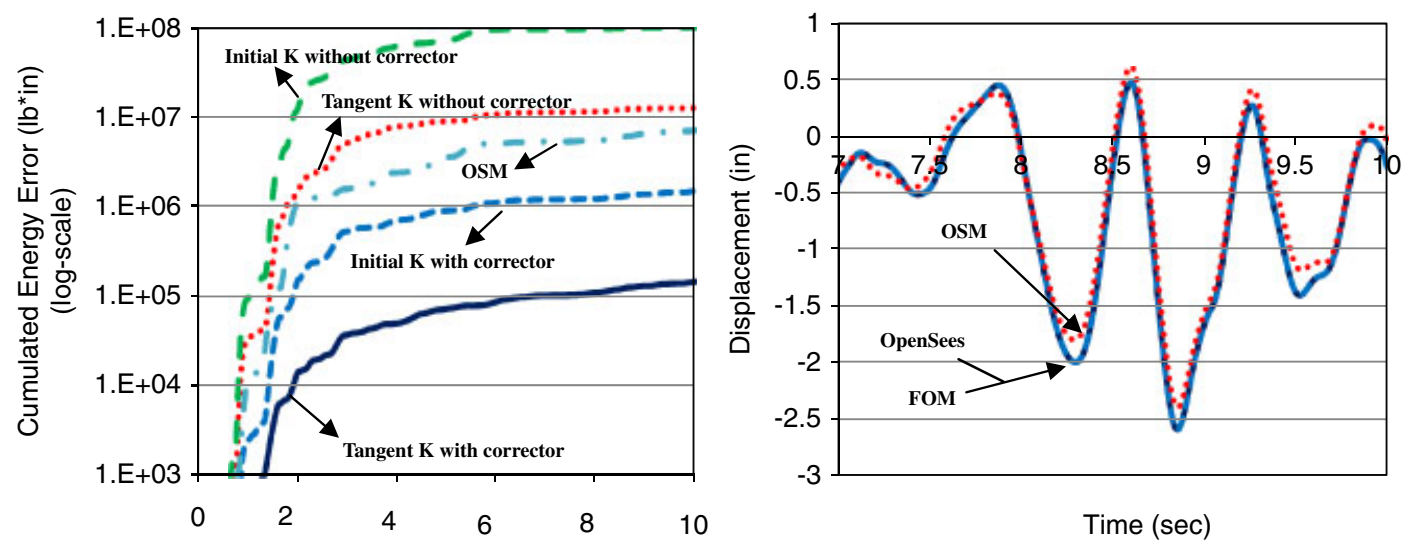

(a)

Time (sec)

(b)

Figure 11. Comparison of shear wall model results computed from various schemes: (a) cumulated energy error, $E_{\mathrm{c}}$ and (b) solutions from OpenSees, FOM and OSM.

that FOM traces the reference solution very well, while OSM deviates from it, especially at the peak points.

The second example is a 6-story shear model with the cross section details shown in Figure 8. The values of the concentrated mass blocks and heights for each floor are displayed in Figure 12. For hybrid simulation purposes, the building is broken up into two parts, the first floor serves as the first substructure and the remaining portion as the second substructure. The entire building is also simulated in OpenSees to provide a reference solution. An incremental time step of $0.005 \mathrm{~s}$ is chosen for this example. 


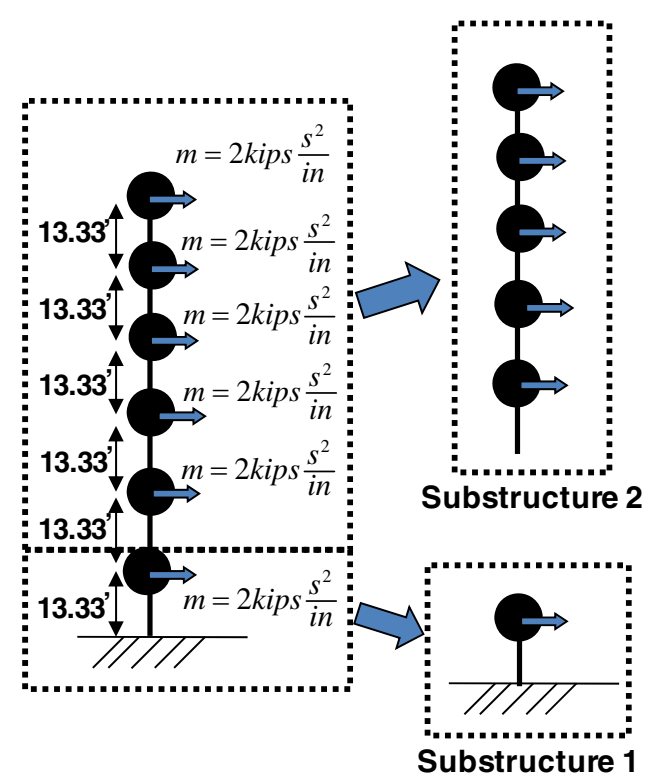

Figure 12. Details of the 6-story model.
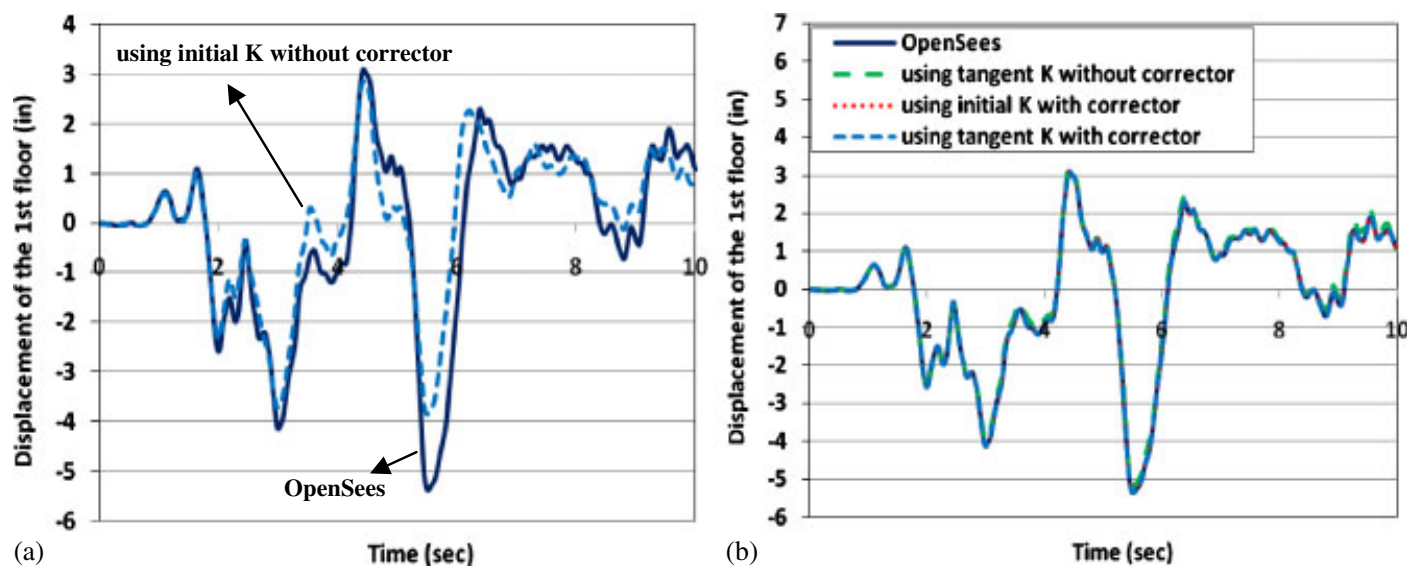

Figure 13. Simulated seismic response of the 6-story model: (a) initial stiffness without the corrector step and (b) results from various FOM schemes.

The same four schemes employed in the SDOF cantilever example above are used in this example as well to show the effect of the various parts and assumptions of FOM. In addition, since all the tangent stiffness updating methods gave close results, only the results from the BFGS method are presented. Figure 13(a) shows the result from the first scheme in which the initial stiffness is used but not the corrector step. Clearly, the solution diverges from the reference solution. The simulation results from the other three schemes are plotted in Figure 13(b), which shows that the 

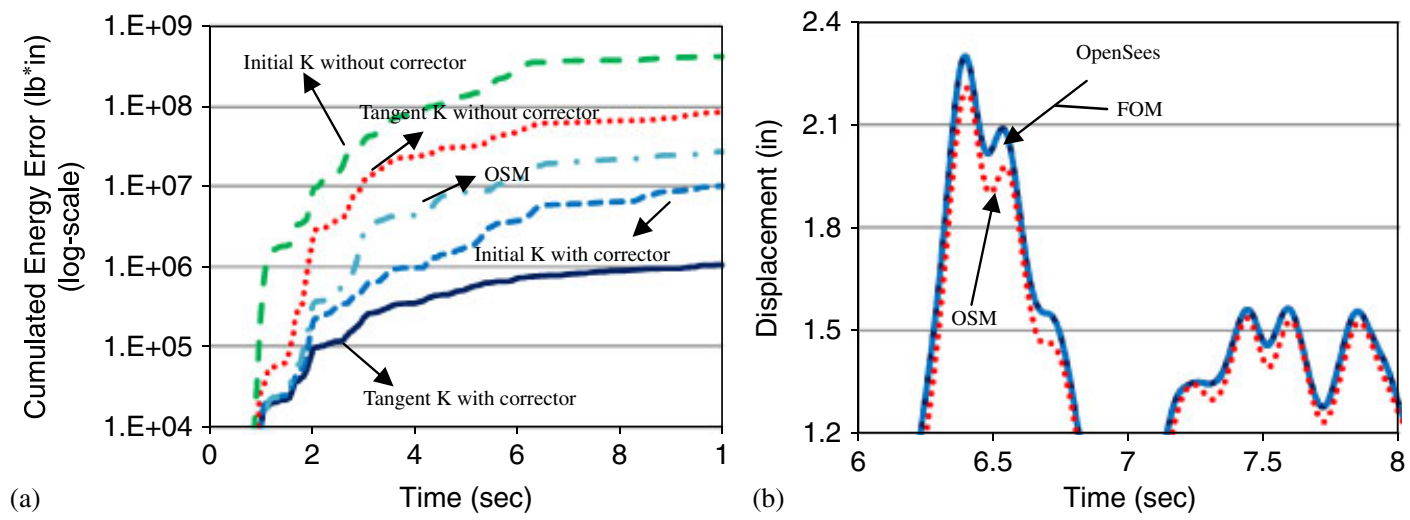

Figure 14. Comparison of 6-story model results computed from various schemes: (a) cumulated energy error, $E_{\mathrm{c}}$ and (b) solutions from OpenSees, FOM and OSM.

simulated seismic responses from these three schemes match the reference solution better than the one in Figure 13(a). However, zooming in on the plot (not shown) shows that there are differences, which can be seen by computing the cumulative energy error, $E_{\mathrm{c}}$. The value of $E_{\mathrm{c}}$ associated with the first-floor displacement is calculated and plotted in Figure 14. As noted in the SDOF cantilever example, the use of the tangent stiffness alone or initial stiffness with corrector both improve the simulation accuracy compared with just using the initial stiffness; in particular, $E_{\mathrm{c}}$ is more than an order of magnitude less in the case when the initial stiffness with corrector is used. As alluded to previously in Figure 11, Figure 14 shows that full implementation of FOM (with tangent stiffness and corrector) results in significantly less errors than application of the traditional OSM.

\section{HANDLING DISPLACEMENT CONTROL ERRORS IN FOM}

Displacement control errors in hydraulic actuators can greatly affect simulation accuracy during hybrid testing. The I-modification technique [18] was proposed as a means to suppress the adverse effects of displacement control errors when the OSM method is used. However, it has been shown in [4] that when the OSM with I-modification is used to simulate structural behavior with significant inelastic behavior, the resulting simulated displacements may suffer in accuracy.

The effect of displacement control errors on the performance of the proposed algorithm is studied using the 6-story shear model. A constant overshoot displacement control error of 0.001 in is assumed in the simulations. Results from FOM are compared with those from OSM with Imodification in Figure 15 to show the benefits of using the new method. It can be seen from the figure that OSM with I-modification deviates from the reference solution from OpenSees. On the other hand, the proposed method is still able to achieve good performance. This is attributed to the fact that the corrector in the proposed method relies entirely on measured information from the tested structures, i.e. the restoring force, instead of using an estimated initial stiffness or tangent stiffness of the structure in the case of OSM. 


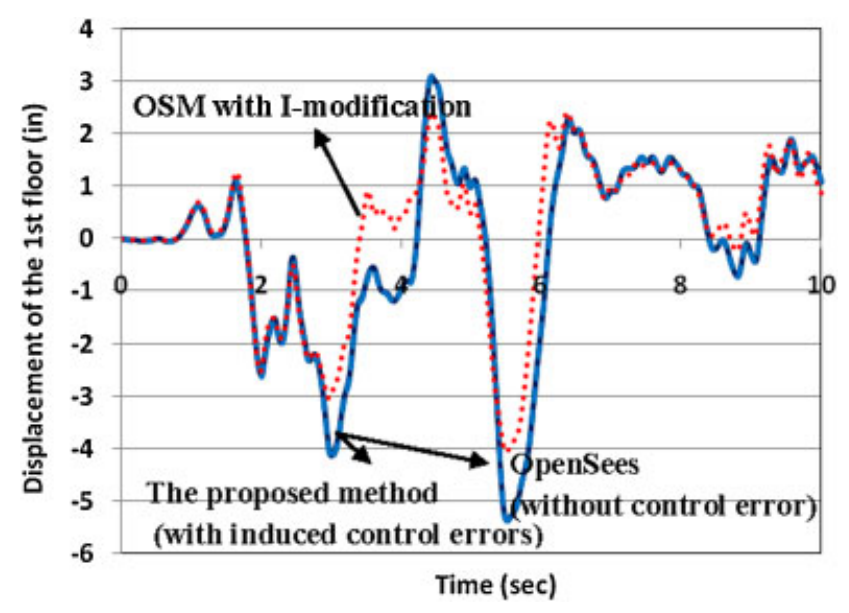

Figure 15. The effect of displacement control error on simulation results.

\section{CONCLUSIONS}

A new hybrid simulation technique was presented in this paper. The technique, termed full operator method, or FOM, differs in two significant ways from the regular OSM. First, FOM increases the accuracy of the predictor step by including a predictor acceleration term and by relying on the tested structure's estimated tangent stiffness. Second, FOM employs the measured restoring force from the tested structure for the corrector step, without resorting to approximations such as those used in OSM. Based on the advantages of the method, it is suggested that FOM is a better candidate for general hybrid simulation than the OSM and other similar techniques. Furthermore, since both predictor displacement and velocity are generated from the algorithm, FOM can be applied to real-time hybrid simulation. The capabilities of FOM were demonstrated through a number of numerical simulations, including an elastic SDOF vibrator, an inelastic SDOF shear wall system, and an inelastic 6-story shear structure. A final exercise showed that FOM can also be used to generate good results even when displacement control errors are present.

The proposed FOM has shown good performance in the numerical simulation exercises presented in this paper. However, it is still necessary to conduct physical tests to make sure that it would work under practical hybrid simulation conditions. Moreover, the developed method has been applied to structures with overall hardening response and dominated by first mode behavior. Additional research is necessary to investigate if it will provide similarly good results for softening structures and for structures with higher mode response. Although no adverse stability issues were noted in this research, more work is needed to formally study the stability characteristics of the method.

\section{ACKNOWLEDGEMENTS}

The research described herein was sponsored in part by the National Science Foundation under Grant No. CMS 0530383 and the University of Michigan. The opinions, findings, and conclusions expressed in this paper are those of the authors and do not necessarily reflect the views of the sponsors. 


\section{REFERENCES}

1. Takanashi K, Udagawa K, Seki M, Okada T, Tanaka K. Earthquake response analysis of steel frames by computer-actuator on-line system. Proceedings of the 5th Japan Earthquake Engineering Symposium, Tokyo, Japan, November 1978; 1321-1328.

2. Takanashi K, Nakashima M. Japanese activities on on-line testing. Journal of Structural Engineering (ASCE) 1987; 113(7):1014-1032.

3. Shing PB, Mahin SA. Pseudodynamic test method for seismic performance evaluation: theory and implementation. Report No. UCB/EERC-84/12, Earthquake Engineering Research Center, University of California, Berkeley, 1984.

4. Combescure D, Pegon P. $\alpha$-Operator splitting time integration technique for pseudodynamic testing error propagation analysis. Soil Dynamics and Earthquake Engineering 1997; 16(7-8):427-443.

5. Mahin SA, Shing PB. Pseudodynamic method for seismic testing. Journal of Structural Engineering (ASCE) 1985; 111(7):1482-1503.

6. Shing PB, Vannan MT, Cater E. Implicit time integration for pseudodynamic tests. Earthquake Engineering and Structural Dynamics 1991; 20:551-576.

7. Ghaboussi J, Yun GJ, Hashash YMA. A novel predictor-corrector algorithm for sub-structure pseudo-dynamic testing. Earthquake Engineering and Structural Dynamics 2006; 35:453-476.

8. Nakashima M, Masaoka N. Real-time on-line test for MDOF systems. Earthquake Engineering and Structural Dynamics 1999; 28:393-420.

9. Wu B, Bao H, Ou J, Tian S. Stability and accuracy analysis of central difference method for real-time substructure testing. Earthquake Engineering and Structural Dynamics 2005; 34:705-718.

10. Darby AP, Blakeborough A, Williams MS. Improved control algorithm for real-time substructure testing. Earthquake Engineering and Structural Dynamics 2001; 30:431-448.

11. Pan P, Tomofuji H, Wang T, Nakashima M, Ohsaki M, Mosalam K. Development of peer-to-peer (P2P) internet online hybrid test system. Earthquake Engineering and Structural Dynamics 2006; 35:867-890.

12. Thewalt CR, Mahin SA. An unconditionally stable hybrid pseudodynamic algorithm. Earthquake Engineering and Structural Dynamics 1994; 24:723-731.

13. Hung CC, El-Tawil S. A method for estimating specimen tangent stiffness for hybrid simulation. Earthquake Engineering and Structural Dynamics 2009; 38:115-134.

14. Zhang Y, Sause R, Ricles JM, Naito CJ. Modified predictor-corrector numerical scheme for real-time pseudo dynamic tests using state-space formulation. Earthquake Engineering and Structural Dynamics 2005; 34:271-288.

15. Shing PB, Vannan MT, Carter E. Implicit time integration for pseudodynamic tests. Earthquake Engineering and Structural Dynamics 1991; 20:551-576.

16. Hilber HM, Hughes TJR, Taylor RL. Improved numerical dissipation for time integration algorithm in structural dynamics. Earthquake Engineering and Structural Dynamics 1977; 5:283-292.

17. Nakashima M, Kaminosono T, Ishida M, Ando K. Integration techniques for substructure pseudo dynamic test. Fourth U.S. National Conference on Earthquake Engineering, Palm Springs, CA, 1990; 515-524.

18. Nakashima M, Kato H. Experimental Error Growth Behavior and Error Growth Control in On-line Computer Test Control Method. Building Research Institute, Ministry of Construction, 1987.

19. Thewalt CR, Roman M. Performance parameters for pseudodynamic tests. Journal of Structural Engineering (ASCE) 1994; 120(9):2768-2781.

20. Igarashi A, Seible F, Hegemeier GA. Development of the pseudodynamic technique for testing a full scale 5-story shear wall structure. Proceedings of U.S.-Japan Seminar on the Development and Future Directions of Structural Testing Techniques, Honolulu, U.S.A., 1990.

21. Carrion JE, Spencer BF. Real-time hybrid testing using model-based delay compensation. Proceedings of the 4th International Conference on Earthquake Engineering, Taipei, Taiwan, 2006.

22. Broyden CG. A class of methods for solving nonlinear simultaneous equations. Mathematics of Computation 1965; 19:577-593.

23. Nocedal J, Wright SJ. Numerical Optimization. Springer: Berlin, 1999. ISBN: 0-387-98793-2.

24. Kwon OS, Nakata N, Park KS, Elnashai A, Spencer B. User Manual and Examples for UI-SIMCOR v2.6 Multi-Site Substructure Pseudo-Dynamic Simulation Coordinator, University of Illinois, Urana-Champaign, 2007.

25. OpenSees version 1.7 User Manual. Pacific Earthquake Engineering Research Center, University of California Berkeley, 2006. Available from: http://opensees.berkeley.edu. 